\title{
Decomposition of Monomial Algebras: Applications and Algorithms
}

\author{
JANKO BÖHM, DAVID EISENBUd, AND MAX J. Nitsche
}

\begin{abstract}
Considering finite extensions $K[A] \subseteq K[B]$ of positive affine semigroup rings over a field $K$ we have developed in [BEN] an algorithm to decompose $K[B]$ as a direct sum of monomial ideals in $K[A]$. By computing the regularity of homogeneous semigroup rings from the decomposition, we have confirmed the Eisenbud-Goto conjecture in a range of new cases not tractable by standard methods. Here we first illustrate this technique and its implementation in our Macaulay2 package MonomialAlgebras by computing the decomposition and the regularity step by step for an explicit example. We then focus on ring-theoretic properties of simplicial semigroup rings. From the characterizations given in [BEN], we develop and prove explicit algorithms testing various properties, including being Buchsbaum, CohenMacaulay, Gorenstein, normal, and seminormal. All algorithms are implemented in our Macaulay2 package.
\end{abstract}

INTRODUCTION. Let $B$ be a positive affine semigroup, that is, $B$ is a finitely generated subsemigroup of $\mathbb{N}^{m}$ for some $m$. Let $K$ be a field and $K[B]$ the affine semigroup ring associated to $B$, which can be identified with the subring of $K\left[t_{1}, \ldots, t_{m}\right]$ generated by monomials $t^{u}:=t_{1}^{u_{1}} \cdots t_{m}^{u_{m}}$, where $u=\left(u_{1}, \ldots, u_{m}\right) \in B$. Denote by $C(B)$ and by $G(B)$ the cone and the group generated by $B$. From now on let $A \subseteq B$ be positive affine semigroups with $C(A)=C(B)$. We will now discuss the decomposition of $K[B]$ into a direct sum of monomial ideals in $K[A]$. Observe that

$$
K[B]=\bigoplus_{g \in G} K \cdot\left\{t^{b} \mid b \in B \cap g\right\},
$$

where $G:=G(B) / G(A)$. We have $C(A)=C(B)$ if and only if $K[B]$ is a finitely generated $K[A]$-module. From this it follows that $G$ is finite, and we can compute the above decomposition since all summands are finitely generated. Moreover, there are shifts $h_{g} \in G(B)$ such that $I_{g}:=K \cdot\left\{t^{b-h_{g}} \mid b \in B \cap g\right\}$ is a monomial ideal in $K[A]$. Thus, $K[B] \cong \bigoplus_{g \in G} I_{g}\left(-h_{g}\right)$ as $\mathbb{Z}^{m}$-graded $K[A]$-modules (with $\operatorname{deg} t^{b}=b$ ). A detailed formulation of the algorithm computing the ideals $I_{g}$ and shifts $h_{g}$ and a more general version of the decomposition in the setup of cancellative abelian semigroup rings over an integral domain can be found in [BEN, Algorithm 1, Theorem 2.1].

Our original motivation for developing this decomposition was to provide a fast algorithm to compute the Castelnuovo-Mumford regularity $\operatorname{reg} K[B]$ of a homogeneous semigroup ring in order to test the Eisenbud-Goto conjecture [EG, Conjecture p. 93]. Recall that the Castelnuovo-Mumford regularity reg $M$ of a finitely generated graded module $M$ over a standard graded polynomial ring $R=K\left[x_{1}, \ldots, x_{n}\right]$ is defined as the smallest integer $m$ such that every $j$-th syzygy module of $M$ is generated by elements of degree at most $m+j$. Moreover, $B$ is called a homogeneous semigroup if there exists a group homomorphism $\operatorname{deg}: G(B) \rightarrow \mathbb{Z}$ with $\operatorname{deg} b_{i}=1$ for $i=1, \ldots, n$, where

2010 Mathematics Subject Classification. 13D45; 13P99, 13H10.

MonomialAlgebras version 2.3. 
$\operatorname{Hilb}(B)=\left\{b_{1}, \ldots, b_{n}\right\}$ is the minimal generating set of $B$; by $\operatorname{reg} K[B]$ we mean its regularity with respect to the $R$-module structure which is given by the $K$-algebra homomorphism $R \rightarrow K[B], x_{i} \mapsto t^{b_{i}}$.

The toric Eisenbud-Goto conjecture can be formulated as follows: let $K$ be a field and $B$ a homogeneous semigroup, then $\operatorname{reg} K[B] \leq \operatorname{deg} K[B]-\operatorname{codim} K[B]$, where $\operatorname{deg} K[B]$ denotes the degree and $\operatorname{codim} K[B]:=\operatorname{dim}_{K} K[B]_{1}-\operatorname{dim} K[B]$ the codimension. Even this special case of the EisenbudGoto conjecture is largely open; for references on known results see [BEN, §4]. The regularity of $K[B]$ is usually computed from a minimal graded free resolution. If $n$ is large this computation is very expensive, and hence it is impossible to test the conjecture systematically in high codimension using this method. However, choosing $A$ to be generated by minimal generators $e_{1}, \ldots, e_{d}$ of $C(B)$ of degree 1 the regularity can be computed as $\operatorname{reg} K[B]=\max \left\{\operatorname{reg} I_{g}+\operatorname{deg} h_{g} \mid g \in G\right\}$, where $\operatorname{reg} I_{g}$ denotes the regularity of $I_{g}$ with respect to the canonical $T=K\left[x_{1}, \ldots, x_{d}\right]$-module structure given by $T \rightarrow K[A], x_{i} \mapsto t^{e_{i}}$. Since the free resolution of every ideal $I_{g}$ appearing has length at most $d-1$, this computation is typically much faster than the traditional approaches. This enabled us to test the conjecture for a large class of homogeneous semigroup rings by using our regularity algorithm. See [BEN, §4] for details.

In first section, we illustrate a step by step decomposition and regularity computation for an explicit example using our Macaulay2 [M2] package MonomialAlgebras. We say that $K[B]$ is a simplicial semigroup ring if the cone $C(B)$ is simplicial. In second section, we focus on simplicial semigroup rings $K[B]$. Based on the characterizations of ring-theoretic properties given in [BEN, Proposition 3.1] we develop explicit algorithms for testing whether $K[B]$ is Buchsbaum, Cohen-Macaulay, Gorenstein, seminormal, or normal. We also discuss that, by known results, all these ring-theoretic properties imply the Eisenbud-Goto conjecture. The algorithms mentioned are implemented in our Macaulay 2 package.

DECOMPOSITION AND REGULARITY. Our Macaulay2 package can be loaded by

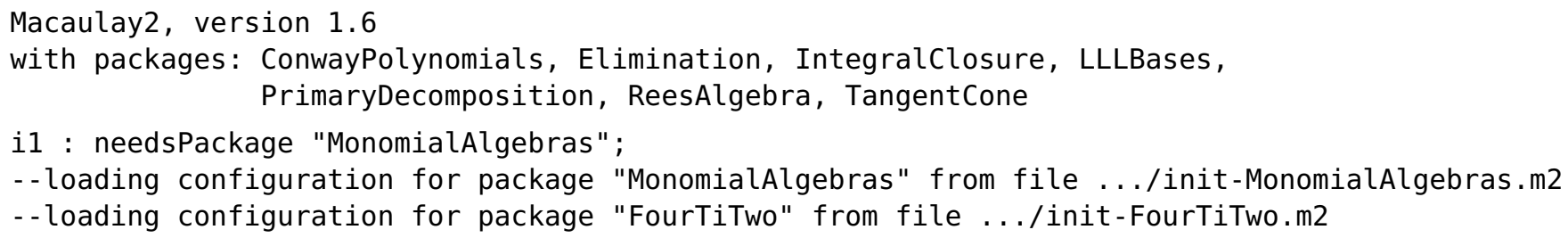

We discuss the decomposition and computation of the regularity at the example of the homogeneous semigroup $B \subset \mathbb{N}^{3}$ specified by a list of generators

i2: $B=\{\{4,0,0\},\{2,2,0\},\{2,0,2\},\{0,2,2\},\{0,3,1\},\{3,1,0\},\{1,1,2\}\}$;

As an input for our algorithms we encode this data in a multigraded polynomial ring

i3 : $K=Z Z / 101$;

i4: $S=K\left[x_{-} 1 \ldots x_{-} 7\right.$, Degrees $\left.=>B\right]$;

The usual approach to computing reg $K[B]$ is to obtain it from a minimal graded free resolution of the toric ideal $I_{B}$ with respect to the standard grading:

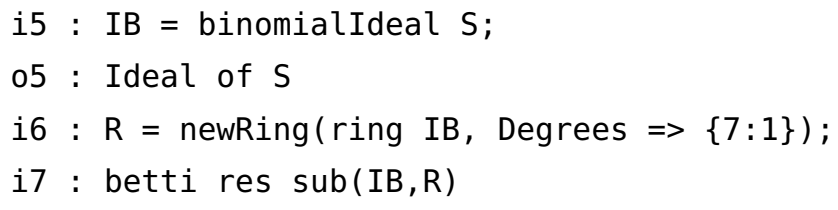




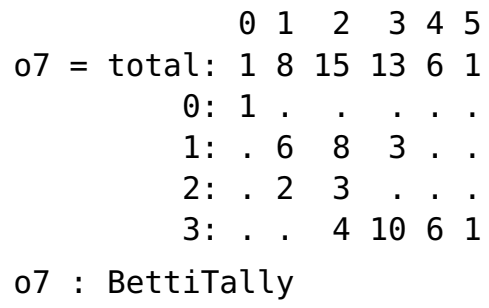

Hence, we observe that $\operatorname{reg} K[B]=3$. On the other hand, using the decomposition of $K[B]$, we can proceed as follows: The command

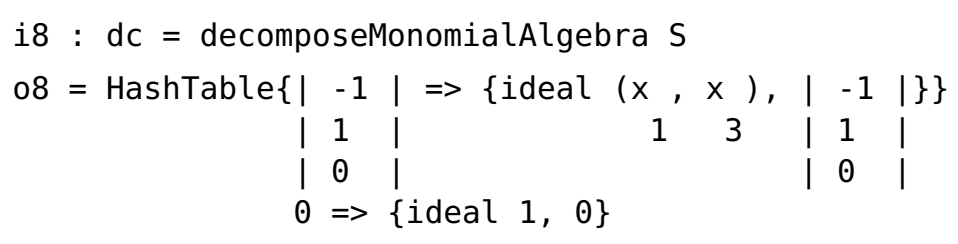

08 : HashTable

decomposes $K[B]$ over $K[A]$ where $A \subseteq B$ is generated by minimal generators of $C(B)$ with minimal coordinate sum; so in the example $A=\langle(4,0,0),(2,2,0),(2,0,2),(0,2,2),(0,3,1)\rangle$. The keys of the hash table represent the elements of $G$ and the values are the tuples $\left(I_{g}, h_{g}\right)$, hence

$$
K[B] \cong\left\langle\bar{x}_{1}, \bar{x}_{3}\right\rangle(-(-1,1,0)) \oplus K[A]
$$

as $\mathbb{Z}^{3}$-graded $K[A]$-modules; here we write $K[A] \cong T / J$ with $T=K\left[x_{1}, x_{2}, x_{3}, x_{4}, x_{5}\right]$ and $\bar{x}_{i}$ for the class of $x_{i}$. The on-screen output of Macaulay 2 does not distinguish between the class and the representative. With respect to the standard grading $\operatorname{deg} u=\left(u_{1}+u_{2}+u_{3}\right) / 4$, Equation ( $\dagger$ ) yields $\operatorname{reg} K[B]=\max \left\{\operatorname{reg}\left\langle\bar{x}_{1}, \bar{x}_{3}\right\rangle+(-1+1+0) / 4, \operatorname{reg} K[A]+0\right\}$. We compute $\operatorname{reg} K[A]$ :

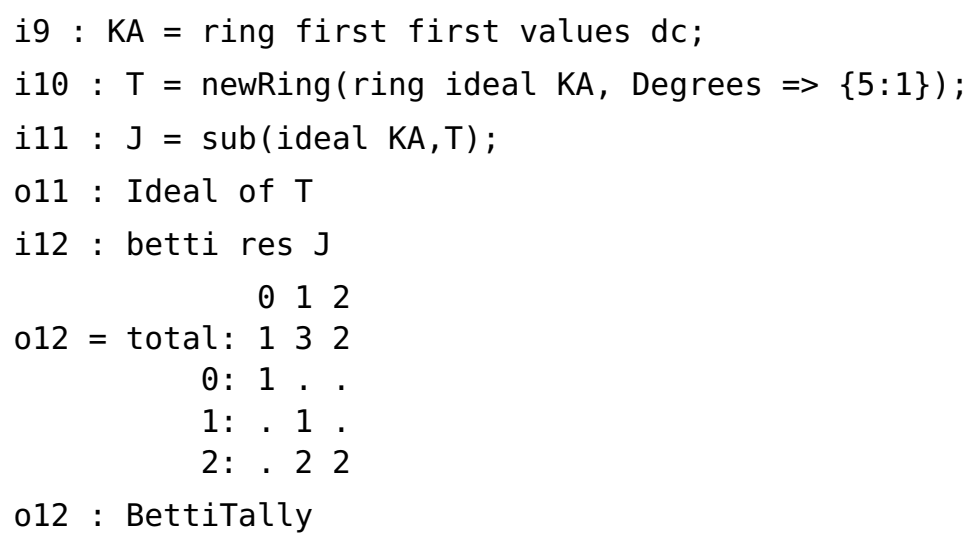

Hence $\operatorname{reg} K[A]=2$. We can compute $\operatorname{reg}\left\langle\bar{x}_{1}, \bar{x}_{3}\right\rangle$ as follows:

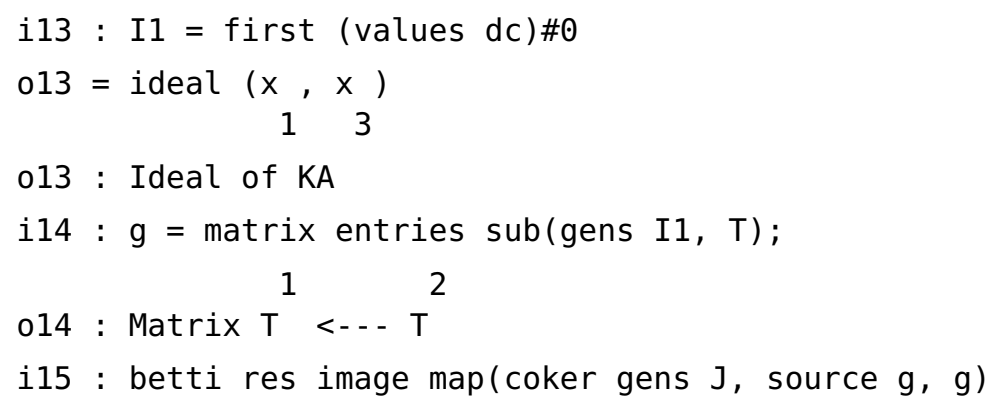




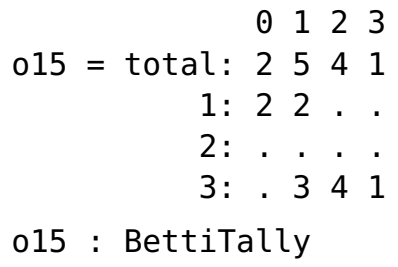

Thus, reg $\left\langle\bar{x}_{1}, \bar{x}_{3}\right\rangle=3$, and therefore we see again that reg $K[B]=3$. Observe that the resolution of $K[B]$ has length 5 , whereas the ideals $I_{g}$ have resolutions of length at most 3 . The command

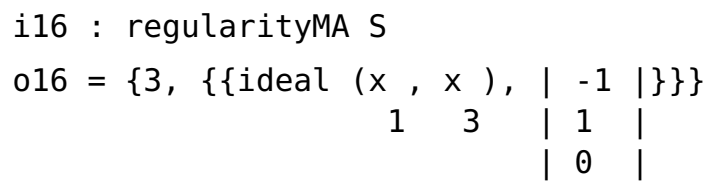

provides an implementation of this approach, also returning the tuples $\left(I_{g}, h_{g}\right)$ where the maximum is achieved. By $[\mathrm{BEN}$, Proposition 4.1] we have $\operatorname{deg} K[B]=\# G \cdot \operatorname{deg} K[A]=10$ since

i17 : degree $\mathrm{J}$

$017=5$

Moreover, $\operatorname{codim} K[B]=4$ since $\operatorname{dim} K[B]=\operatorname{dim} C(B)=3$. Hence the $\operatorname{ring} K[B]$ satisfies the EisenbudGoto bound.

AlgORITHMS FOR RING THEORETIC PROPERTIES. In this section, we focus on simplicial semigroup rings $K[B]$. Based on the characterizations given in [BEN, Proposition 3.1], we develop and prove explicit algorithms for testing whether $K[B]$ is Buchsbaum, Cohen-Macaulay, Gorenstein, seminormal, or normal. In the simplicial case, all these properties are independent of $K$ and they imply the Eisenbud-Goto conjecture by results of [SV, Corollary p. 307], [T, Corollary 2.3 and Proposition 2.2], and [N, Theorem 3.16]. As an example, consider the following homogeneous simplicial semigroup $B \subset \mathbb{N}^{3}$ specified by the generators

$$
\text { i18: } B=\{\{4,0,0\},\{0,4,0\},\{0,0,4\},\{1,0,3\},\{0,2,2\},\{3,0,1\},\{1,2,1\}\} ;
$$

We compute the decomposition of $K[B]$ over $K[A]$, where $A=\langle(4,0,0),(0,4,0),(0,0,4)\rangle \subset B$ is generated again by minimal generators of $C(B)$ with minimal coordinate sum.

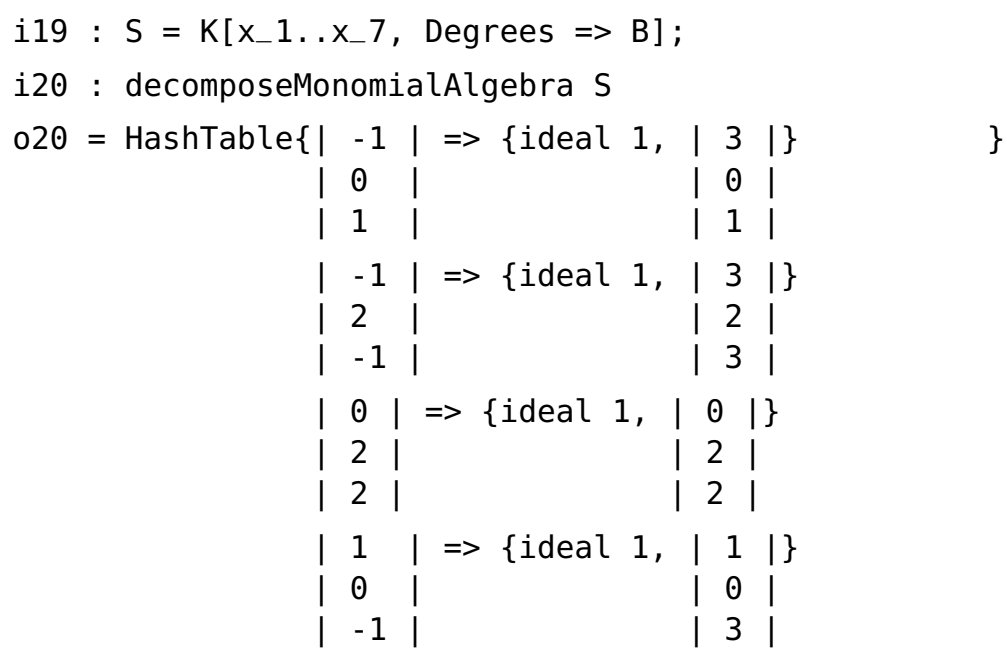




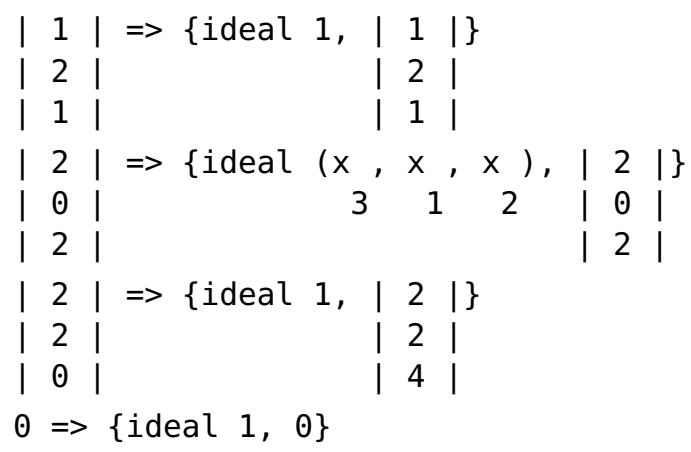

020 : HashTable

Hence, $K[B] \cong K[A] \oplus K[A](-1)^{4} \oplus K[A](-2)^{2} \oplus\left\langle x_{1}, x_{2}, x_{3}\right\rangle(-1)$ with respect to the standard grading induced by $\operatorname{deg} u=\left(u_{1}+u_{2}+u_{3}\right) / 4$. It follows that depth $K[B]=1$; thus, $K[B]$ is not Cohen-Macaulay. Hence, $K[B]$ is also not normal by $[\mathrm{H}$, Theorem 1]. We can test seminormality via Algorithm 1.

Algorithm 1 (Seminormality test).

Input: A simplicial semigroup $B \subseteq \mathbb{N}^{m}$.

Output: true if $K[B]$ is seminormal, false otherwise.

1: Let $e_{1}, \ldots, e_{d} \in B$ be minimal generators of $C(B)$ with minimal coordinate sum, and set $A:=\left\langle e_{1}, \ldots, e_{d}\right\rangle$.

2: Compute $B_{A}:=\{x \in B \mid x \notin B+(A \backslash\{0\})\}$ as described in [BEN, Algorithm 1, Step 1].

3: for all $x \in B_{A}$ do

4: $\quad$ Solve the linear system of equations $\sum_{i=1}^{d} \lambda_{i} e_{i}=x$ for $\lambda=\left(\lambda_{1}, \ldots, \lambda_{d}\right) \in \mathbb{Q}^{d}$.

5: $\quad$ if $\|\lambda\|_{\infty}>1$ then return false.

6: end for

7: return true.

Here, $\|-\|_{\infty}$ denotes the maximum norm. Note that all $\lambda_{i}$ are non-negative since $C(B)$ is a simplicial cone. Verifying in Step 5 the condition $\|\lambda\|_{\infty} \geq 1$ instead, results in an algorithm which tests normality. Using our package we observe that, in the example above, $K[B]$ is not seminormal:

i21 : isSeminormalMA B

$021=$ false

The Buchsbaum property can be tested by Algorithm 2. We denote by $K[A]_{+}$the homogeneous maximal ideal of $K[A]$.

Algorithm 2 (Buchsbaum test).

Input: A simplicial semigroup $B=\left\langle b_{1}, \ldots, b_{n}\right\rangle \subseteq \mathbb{N}^{m}$.

Output: true if $K[B]$ is Buchsbaum, false otherwise.

1: Let $e_{1}, \ldots, e_{d} \in B$ be minimal generators of $C(B)$ with minimal coordinate sum, and set $A:=\left\langle e_{1}, \ldots, e_{d}\right\rangle$.

2: Using the (minimal) generators $e_{1}, \ldots, e_{d}$ of $A$ decompose $K[B] \cong \bigoplus_{g \in G} I_{g}\left(-h_{g}\right)$, where $I_{g} \subseteq K[A], h_{g} \in G(B)$ and $G=G(B) / G(A)$ by [BEN, Algorithm 1].

3: if there exists $g \in G$ with $I_{g} \neq K[A]$ and $I_{g} \neq K[A]_{+}$then return false.

4: $H:=\left\{h_{g} \mid g \in G\right.$ with $\left.I_{g}=K[A]_{+}\right\}$.

5: $C:=\left\{b_{1}, \ldots, b_{n}\right\} \backslash\left\{0, e_{1}, \ldots, e_{d}\right\}$. 
6: $H+C:=\left\{h_{g}+b_{i} \mid h_{g} \in H, b_{i} \in C\right\}$.

7: return true if $(H+C) \cap H=\varnothing$ and false otherwise.

Proof of Algorithm 2. By [BEN, Proposition 3.1], the ring $K[B]$ is Buchsbaum iff each ideal $I_{g}$ is either equal to $K[A]$, or to $K[A]_{+}$and $h_{g}+b \in B$ for all $b \in \operatorname{Hilb}(B)$. So, Step 3 is correct and we may now assume that $I_{g}=K[A]$ or $I_{g}=K[A]_{+}$for all $g \in G$. Recall that $I_{g}=\left\{t^{\nu-h_{g}} \mid v \in \Gamma_{g}\right\} K[A]$ where $\Gamma_{g}=\left\{x \in B_{A} \mid x \in g\right\}$. Moreover, $\left\{t^{\nu-h_{g}} \mid v \in \Gamma_{g}\right\}$ is always a minimal generating set of $I_{g}$ and $h_{g}=\sum_{k=1}^{d} \min \left\{\lambda_{k}^{v} \mid v \in \Gamma_{g}\right\} e_{k}$ where $v=\sum_{k=1}^{d} \lambda_{k}^{v} e_{k}$ with $\lambda_{k}^{v} \in \mathbb{Q}$. Since $h+e_{k} \in B_{A}$ for all $h \in H$ and all $k=1, \ldots, d$, we have $H \cap B=\varnothing$. In case that $(H+C) \cap H \neq \varnothing$, we obtain $h+b \notin B$ for some $h \in H$ and some $b \in B \backslash\{0\}$, that is $h+\operatorname{Hilb}(B) \nsubseteq B$. Hence, $K[B]$ is not Buchsbaum.

In case that $K[B]$ is not Buchsbaum, there is an $h \in H$ and some $b \in \operatorname{Hilb}(B)$ such that $h+b \notin B$. It is now sufficient to show that $b \in C$ and $h+b \in H$. By the above argument, $b \in C$. Let $m_{k}=h+b+e_{k}$ for $k=1, \ldots, d$. Suppose that $m_{i} \notin B_{A}$ for some $i \in\{1, \ldots, d\}$. Since $m_{k}-e_{k} \notin B$ for all $k=1, \ldots, d$, necessarily $m_{i}-e_{j} \in B$ for some $j \neq i$. Consider $y=m_{j}-\sum_{k=1}^{d} n_{k} e_{k} \in B$ with $n_{k} \in \mathbb{N}$ such that $\sum_{k=1}^{d} n_{k}$ is maximal. By construction $y \in B_{A}$, moreover, $n_{j}=0$ since $m_{j}-e_{j} \notin B$. In the same way if $x=m_{i}-e_{j}-\sum_{k=1}^{d} n_{k} e_{k} \in B$ with $\sum_{k=1}^{d} n_{k}$ maximal, then $x \in B_{A}$. Since $m_{i}, m_{j} \in g$ for some $g \in G$, we also have $x, y \in g$. Since $e_{1}, \ldots, e_{d}$ are linearly independent, we have $\lambda_{j}^{y}-\lambda_{j}^{x} \geq 2$. Moreover, since $t^{y-h_{g}}, t^{x-h_{g}} \in K[A]$ we get that $t^{y-h_{g}}$ is not a linear form. Hence $I_{g} \neq K[A]$ and $I_{g} \neq K[A]_{+}$, thus, $m_{k} \in B_{A}$ for all $k=1, \ldots, d$. We have $\# \Gamma_{g} \in\{1, d\}$ by minimality, hence $\Gamma_{g}=\left\{m_{1}, \ldots, m_{d}\right\}$. By construction, $h_{g}=h+b$ and $I_{g}=K[A]_{+}$, therefore $h+b \in H$.

In Step 2 of Algorithm 2, the shifts $h_{g}$ and hence the ideals $I_{g}$ are uniquely determined since $e_{1}, \ldots, e_{d}$ are linearly independent. This is not true for arbitrary generating sets. Continuing the example, by [SV, Corollary p. 307] and

i22 : isBuchsbaumMA B

$022=$ true

it follows that $K[B]$ satisfies the Eisenbud-Goto conjecture. We can also read off from the decomposition the regularity and the Eisenbud-Goto bound: we have $\operatorname{reg} K[A]=0$ and $\operatorname{reg}\left\langle x_{1}, x_{2}, x_{3}\right\rangle=1$, therefore $\operatorname{reg} K[B]=\max \{0,1,2,1+1\}=2$. Moreover, $\operatorname{deg} K[B]$ is the number of ideals which occur in the decomposition, hence $\operatorname{deg} K[B]-\operatorname{codim} K[B]=8-4=4$.

When $B$ is Buchsbaum, the regularity of $K[B]$ is independent of the field $K$ since all ideals in the decomposition are equal to the homogeneous maximal ideal or to $K[A]$.

We finish this section by providing Algorithm 3 for testing the Gorenstein property.

Algorithm 3 (Gorenstein test).

Input: A simplicial semigroup $B \subseteq \mathbb{N}^{m}$.

Output: true if $K[B]$ is Gorenstein, false otherwise.

1: Let $e_{1}, \ldots, e_{d} \in B$ be minimal generators of $C(B)$ with minimal coordinate sum, and set $A:=\left\langle e_{1}, \ldots, e_{d}\right\rangle$.

2: Using the (minimal) generators $e_{1}, \ldots, e_{d}$ of $A$ decompose $K[B] \cong \bigoplus_{g \in G} I_{g}\left(-h_{g}\right)$ where $I_{g} \subseteq K[A]$, $h_{g} \in G(B)$ and $G=G(B) / G(A)$ by [BEN, Algorithm 1].

3: if there exists $g \in G$ with $I_{g} \neq K[A]$ then return false.

4: $H:=\left\{h_{g} \mid g \in G\right\}$.

5: if $h \in H$ with maximal coordinate sum is not unique then return false. 
6: Let $h \in H$ with maximal coordinate sum.

7: while $H \neq \varnothing$ do

8: $\quad$ Let $h_{g} \in H$.

9: $\quad$ if $h-h_{g} \notin H$ then return false.

10: $H:=H \backslash\left\{h_{g}, h-h_{g}\right\}$.

11: end while

12: return true.

Proof of Algorithm 3. By [BEN, Proposition 3.1] the ring $K[B]$ is Gorenstein if and only if $I_{g}=K[A]$ for all $g \in G$ and $H$ has a unique maximal element with respect to $\leq$ given by $x \leq y$ if there is a $z \in B$ such that $x+z=y$. Note that $H=B_{A}$ since $I_{g}=K[A]$ for all $g \in G$. If there is a maximal element $h \in H$, then this element has maximal coordinate sum. If $H$ has more than one element with maximal coordinate sum, then $H$ does not have a unique maximal element. To complete the proof we need to show that an element $h_{g} \in H$ satisfies $h_{g} \leq h$ iff $h-h_{g} \in H$. But this follows from the fact that if $x \notin B_{A}$ then $x+y \notin B_{A}$ for all $x, y \in B$.

Performing Steps 1-3 of Algorithm 3 (and returning true afterwards) also gives a test for the Cohen-Macaulay property.

\section{REFERENCES.}

[BEN] J. Böhm, D. Eisenbud, and M.J. Nitsche, Decomposition of semigroup algebras, Exp. Math. 21 (2012), no. 4, 385-394.

[EG] D. Eisenbud and S. Goto, Linear free resolutions and minimal multiplicity, J. Algebra 88 (1984), no. 1, 89-133.

[M2] D.R. Grayson and M.E. Stillman, Macaulay2, a software system for research in algebraic geometry, available at www. math. uiuc. edu/Macaulay2/.

$[\mathrm{H}]$ M. Hochster, Rings of invariants of tori, Cohen-Macaulay rings generated by monomials, and polytopes, Ann. of Math. (2) 96 (1972), 318-337.

[N] M.J. Nitsche, Castelnuovo-Mumford regularity of seminormal simplicial affine semigroup rings, J. Algebra 368 (2012), 345-357.

[SV] J. Stückrad and W. Vogel, Castelnuovo bounds for locally Cohen-Macaulay schemes, Math. Nachr. 136 (1988), 307-320.

[T] R. Treger, On equations defining arithmetically Cohen-Macaulay schemes. I, Math. Ann. 261 (1982), no. 2, 141-153.

RECEIVED : 2012-06-15_REVISED : 2013-02-27_ACCEPTED : 2013-04-07

boehm@mathematik.uni-kl.de : Fachbereich Mathematik, TU Kaiserslautern, 67663 Kaiserslautern, Germany.

de@ms ri. org : Department of Mathematics, University of California, Berkeley, CA 94720, USA.

nitsche@mis.mpg . de : Max Planck Institute for Mathematics in the Sciences, Inselstrasse 22, 04103 Leipzig, Germany. 